\title{
The BRITE-Constellation Nanosatellite Space Mission And Its First Scientific Results ${ }^{\star}$
}

\author{
G. Handler ${ }^{1, \star \star}$, A. Pigulski ${ }^{2}$, W. W. Weiss ${ }^{3}$, A. F. J. Moffat ${ }^{4}$, R. Kuschnig ${ }^{3,5}$, G. A. Wade ${ }^{6}$, P. Orleański ${ }^{7}$, S. M.
} Ruciński $^{8}$, O. Koudelka ${ }^{5}$, R. Smolec ${ }^{1}$, K. Zwintz ${ }^{9}$, J. M. Matthews ${ }^{10}$, A. Popowicz ${ }^{11}$, D. Baade ${ }^{12}$, C. Neiner $^{13}, A . A$. Pamyatnykh ${ }^{1}$, J. Rowe ${ }^{14}$, and $A$. Schwarzenberg-Czerny ${ }^{1}$

${ }^{1}$ Nicolaus Copernicus Astronomical Center, Bartycka 18, 00-716 Warsaw, Poland

${ }^{2}$ Instytut Astronomiczny, Uniwersytet Wrocławski, ul. Kopernika 11, 51-622 Wrocław, Poland

${ }^{3}$ Institute for Astrophysics, Universität Wien, Türkenschanzstrasse 17, A-1180 Wien, Austria

${ }^{4}$ Départment de physique, Université de Montréal, C. P. 6128, Succ. Centre-Ville, Montréal, QC H3C 3J7, Canada

${ }^{5}$ Institut für Kommunikationsnetze und Satellitenkommunikation, Inffeldgasse 12/l, 8010 Graz, Austria

${ }^{6}$ Department of Physics, Royal Military College of Canada, PO Box 17000, Stn Forces, Kingston, ON K7K 7B4, Canada

${ }^{7}$ Centrum Badań Kosmicznych, Polska Akademia Nauk, Bartycka 18A, 00-716 Warszawa, Poland

${ }^{8}$ Department of Astronomy and Astrophysics, University of Toronto, 50 St. George Street, Toronto, ON M5S 3H4, Canada

${ }^{9}$ Institut für Astro- und Teilchenphysik, Universität Innsbruck, Technikerstrasse 25/8, 6020, Innsbruck, Austria

${ }^{10}$ Department of Physics and Astronomy, University of British Columbia, Vancouver, BC V6T1Z1, Canada

${ }^{11}$ Silesian University of Technology, Institute of Automatic Control, Gliwice, Akademicka 16, Poland

${ }^{12}$ European Organisation for Astronomical Research in the Southern Hemisphere, Karl-Schwarzschild-Str. 2, 85748 Garching b. München, Germany

${ }^{13}$ LESIA, Observatoire de Paris, PSL Research University, CNRS, Sorbonne Universités, UPMC Univ. Paris 06, Univ. Paris Diderot, Sorbonne Paris Cité, 5 place Jules Janssen, F-92195 Meudon, France

${ }^{14}$ Institut de recherche sur les exoplanétes, iREx, Département de physique, Université de Montréal, Montréal, QC, H3C 3J7, Canada

\begin{abstract}
The BRIght Target Explorer (BRITE) Constellation is the first nanosatellite mission applied to astrophysical research. Five satellites in low-Earth orbits perform precise optical two-colour photometry of the brightest stars in the night sky. BRITE is naturally well suited for variability studies of hot stars. This contribution describes the basic outline of the mission and some initial problems that needed to be overcome. Some information on BRITE data products, how to access them, and how to join their scientific exploration is provided. Finally, a brief summary of the first scientific results obtained by BRITE is given.
\end{abstract}

\section{Introduction}

A BRIght Target Explorer (BRITE) is a nanosatellite designed as a cube of $20 \times 20 \times 20 \mathrm{~cm}$ edge length that weighs approximately $7 \mathrm{~kg}$. It carries a $3-\mathrm{cm}$ telescope with a wide field of view (about $24^{\circ}$ on the sky, nearly unvignetted) that feeds an uncooled $4008 \times 2672$-pixel KAI$11002 \mathrm{M}$ CCD. As such, it is predestined to obtain highprecision space photometry of apparently bright stars.

There is more than one BRITE. Each of the partner countries participating in the mission, Austria, Canada, and Poland, funded two satellites. One of each pair is equipped with a blue-sensitive, the other with a red-

\footnotetext{
${ }^{\star}$ Based on data collected by the BRITE Constellation satellite mission, designed, built, launched, operated and supported by the Austrian Research Promotion Agency (FFG), the University of Vienna, the Technical University of Graz, the Canadian Space Agency (CSA), the University of Toronto Institute for Aerospace Studies (UTIAS), the Foundation for Polish Science \& Technology (FNiTP MNiSW), and National Science Centre (NCN).

${ }^{\star \star}$ e-mail: gerald@camk.edu.pl
}

sensitive filter. The passbands are close to JohnsonCousins $B$ and $R_{c}$. The whole ensemble of satellites is called BRITE-Constellation and is therefore capable of multicolour time-resolved photometry.

The six satellites were launched into orbit between February 2013 and August 2014. One of the Canadian satellites, BRITE-Montreal, went astray during launch as it apparently did not separate from the last stage of the rocket. The remaining five satellites are called BRITE-Austria (BAb) and Uni-BRITE (UBr, both Austrian), BRITE-Lem (BLb) and BRITE-Heweliusz (BHr, both Polish), and BRITE-Toronto (BTr, Canadian), and are operating. The abbreviations of the satellites' names, that will be used in what follows, originate from their designations followed by the filter they carry, red (r) or blue (b).

More detailed accounts about the basic goals and outline of the BRITE-Constellation mission have been given by [1] and [2]. In this contribution, we briefly summarize these articles and the first science results, but also provide a glimpse into BRITE operations for prospective users. 


\section{Scientific goals and observations}

As mentioned in Sect. 1, BRITE-Constellation obtains high-precision time-resolved two-colour photometry of bright stars. Apparently bright stars are often intrinsically luminous, which means that the stellar sample accessible to BRITE contains an overrepresentation of hot and evolved stars. As hot stars are short-lived, they are concentrated near the Galactic plane. This area is of prime interest for BRITE observations, because many target stars and bright guide stars used for tracking can be acquired. The primary science goal of the mission is thus easy to define: to study the variability of hot stars, be it caused by pulsation, rotation, wind variations, star-disk interactions, binarity etc. An HR Diagram of the stars observable by BRITE is shown in [1].

The observing strategy itself bears in mind that the longer the time base of observations, the more information on stellar variability can be obtained. Consequently, each BRITE observing run attempts to observe a given field as long as possible, for up to half a year. This goal is met in most cases. Given the low-Earth orbit of the satellites, occultations of the target field by the Earth or stray light imply that only a fraction of the $\sim 100$-min orbital period (up to $40 \%$, depending on the satellite) can be used for observations.

The ensemble of satellites can be used to observe one field with all five of them (diminishing aliases at the satellites' orbital periods), or their number is split between two fields. A given satellite may also observe more than one field during its orbit. In the first few years of operation, it has been found most useful that two pairs of satellites (one with a blue filter, the other with a red one) each observe two different fields separated by about $6 \mathrm{~h}$ in right ascension, with the remaining fifth satellite used as a supplement.

The measurements of BRITE-Constellation are being retrieved by three ground stations, one in Graz (Austria), one in Warsaw (Poland), and the third in Toronto (Canada). Each country operates their satellites largely independently, with occasional help by the partners if need be. The downlink capacity is limited (also because of some interference issues at the European ground stations, see [2]), and therefore full-frame images are generally not downloaded. Instead, windows that contain target stars and a sufficiently large area of blank sky around them are downloaded for further processing. An overview of the BRITE observations so far is presented in Table 1, and the observed fields are depicted on a sky map in Figure 1.

As soon as the first science images had been obtained by the satellites launched earliest, it became clear that the CCDs suffered from radiation damages, causing hot pixels and columns, as well as Charge Transfer Inefficiency (CTI). Obviously, these defects would have a negative influence on the quality of the photometry to be obtained and needed to be mitigated. This was accomplished in three ways. First, some of the satellites were still on Earth when the problem was noticed. Therefore, BHr and BTr were equipped with better shielding, strongly decreasing the radiation damage. Second, a sophisticated data reduction
Table 1: Target fields observed by BRITE-Constellation so far. Data taken from http://brite.craq-astro.ca/doku.php. The total number of stars observed is not the sum of the individual ones as some stars/fields have been re-observed.

\begin{tabular}{lccrr}
\hline Field & $\begin{array}{c}\text { from } \\
\text { civil date }\end{array}$ & $\begin{array}{c}\text { to } \\
\text { civil date }\end{array}$ & $\begin{array}{r}\Delta \mathrm{T} \\
(\mathrm{d})\end{array}$ & \#stars \\
& 01.12 .2013 & 18.03 .2014 & 108 & 15 \\
Ori I & 25.03 .2014 & 18.08 .2014 & 147 & 32 \\
Cen & 29.04 .2014 & 06.09 .2014 & 42 & 19 \\
Sgr I & 12.06 .2014 & 25.11 .2014 & 167 & 36 \\
Cyg I & 02.09 .2014 & 18.02 .2015 & 170 & 37 \\
Per & 24.09 .2014 & 17.03 .2015 & 175 & 35 \\
Orion II & 11.12 .2014 & 28.05 .2015 & 169 & 52 \\
Vel/Pup & 18.03 .2015 & 31.08 .2015 & 167 & 26 \\
Sco & 01.06 .2015 & 25.11 .2015 & 178 & 34 \\
Cyg II & 23.08 .2015 & 17.10 .2015 & 55 & 25 \\
Cas/Cep & 28.10 .2015 & 14.04 .2016 & 180 & 32 \\
CMa/Pup & 18.01 .2016 & 22.07 .2016 & 183 & 40 \\
Cru/Car & 22.01 .017 & 162 & 17 \\
Sgr II & 15.04 .2016 & 23.09 .2016 & 167 \\
Cyg/Lyr & 28.04 .2016 & 21.10 .2016 & 177 & 15 \\
\hline Total & & & & 350 \\
\hline
\end{tabular}

pipeline has been developed that eliminates these adverse effects to a large extent [3].

Third, and most importantly, a "nodding" technique has been implemented in the observations, starting from early 2015. The BRITE satellites no longer stare at the same position. Instead, they are moved back and forth by about $0.2^{\circ}$ every $\approx 20 \mathrm{~s}$. Thus, the stellar images fall onto alternatingly different parts of their CCD windows, whereas the compromised pixels remain the same over such short time intervals. Consequently, differential images are computed, which are almost free of artifacts, and precise photometry can be obtained from these images. We refer to [2] for more information and illustrations of the process. Nodding mode is now the standard observing mode for BRITE. It also guarantees that the Constellation can be operated for much longer than the projected lifetime of two years because radiation damage of the CCDs is now a minor issue in this regard.

\section{BRITE data products and policies}

After the CCD data have been processed through the BRITE photometry pipeline [3], [4], the data for individual stars are assigned to a so-called "contact PI" who is responsible for their final reduction, analysis and publication. This assignment is based on calls for proposals in the years 2008, 2011 and 2016 (still open at the time of writing, see http://www.brite-constellation.at/).

If a given star has been proposed for observation by several researchers, these decide between themselves who will become contact PI. A contact PI is expected to provide a first report on data analysis three months after receiving the data (including a judgement whether or not 


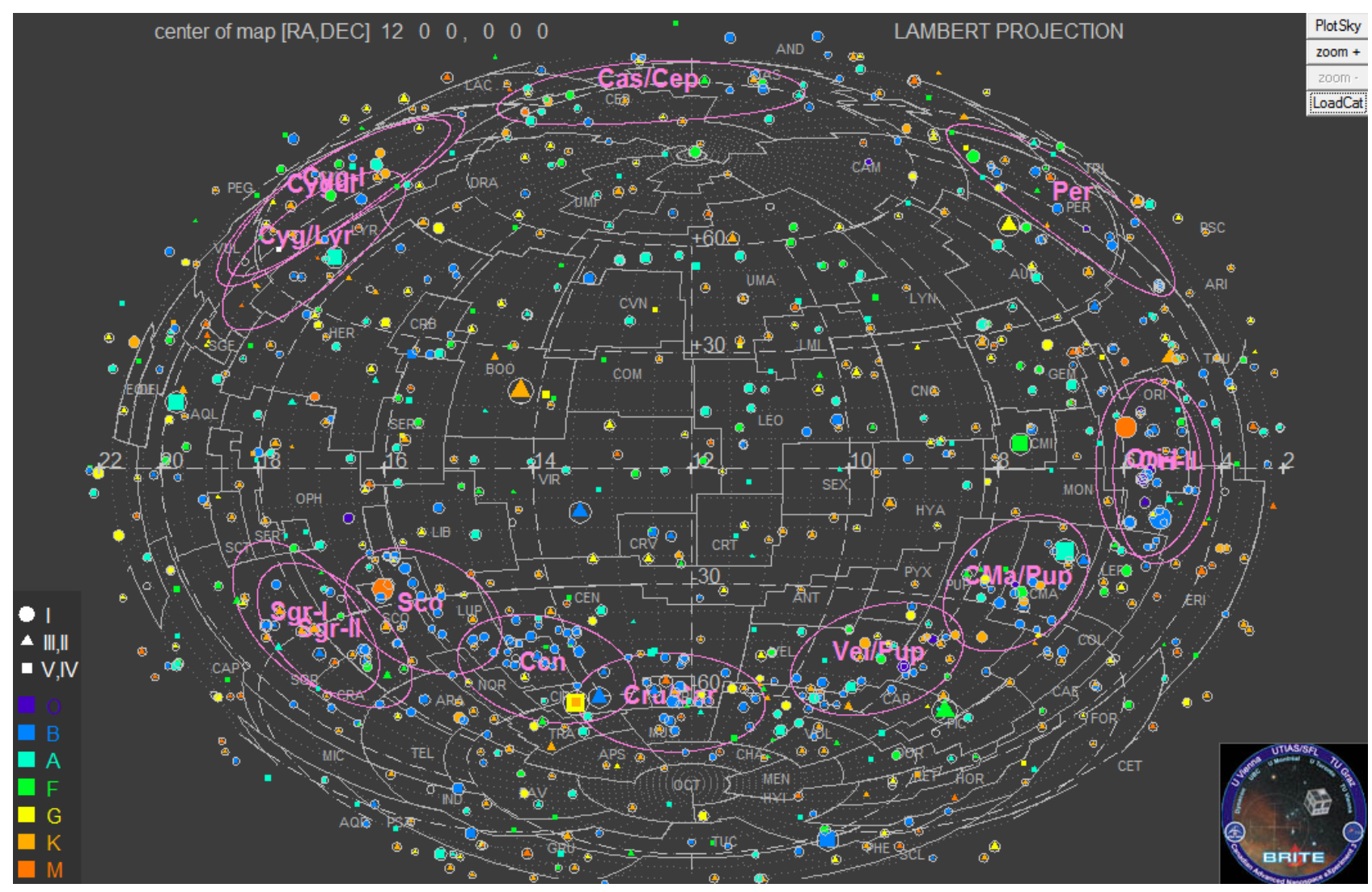

Figure 1: A sky map containing all stars brighter than $V=4.5$. The colour code refers to spectral type, the symbols to luminosity class. Encircled stars have been proposed for BRITE observations. The sky areas labeled and encircled in pink have been observed by BRITE to date (cf. Table 1). Almost the whole galactic plane has been sampled.

the respective data set is suitable for stand-alone publication), and a final report after one year. After this oneyear proprietary period has passed, the data will become public, unless an extension of the proprietary period of up to half a year has been approved in exceptional cases. A list of fields observed, stars measured therein, the respective contact PIs, and links to public data can be found at http://brite.craq-astro.ca/doku.php. Even if a data set is still in the proprietary phase, the contact PI can be approached for possible collaborations, as each contact PI is free to set up their own network of co-workers.

Light curves are supplied to scientists in the form of ASCII files. In the current format, these contain the timing in HJD, the measured target flux, and several additional parameters needed to decorrelate the light curves from remaining instrumental effects. These comprise the $\mathrm{x} / \mathrm{y}$ position of the barycentre of the stellar PSF, the CCD temperature, and three further parameters describing the shape of the PSF (explained in detail by [4]).

Decorrelation of the BRITE photometry is required because the data orginate from an uncooled instrument (CCD and optics) and there is no possibility to acquire calibration images, such as bias, dark and flat field frames. Examples of the temperature changes occuring during a run on a given field, and within the orbit, are again given by [2]. Decorrelation with temperature is usually most important, as the individual CCD pixels' sensitivities depend on it, followed by decorrelation with $\mathrm{x} / \mathrm{y}$ position to compensate for pixel-by-pixel sensitivity variations. Since temperature changes also change the PSF, decorrelation with the three PSF-related parameters may also be necessary, and sometimes the decorrelations must be performed in data subsets depending on average CCD temperature. In rare cases, stray light corrections need to be applied as well.

Several different approaches have been developed to carry out this decorrelation (e.g., [5], [6]). Sometimes it is necessary to first remove a target's variability from the observations to decorrelate optimally [7]). Even though these procedures may appear difficult, and several iterations may be required, the problems are understood and improved algorithms are permanently being developed.

Regarding the organization of the project, the scientific decisions such as target field selection, proposal evaluation, proprietary period extensions etc., are made by the BRITE Executive Science Team (BEST) whose current representatives are also the authors of the present manuscript. The scientific user community is organized as the BRITE-Constellation International Advisory Team (BIAST). Membership is granted after an informal request to one of us (GAW, Gregg.Wade@rmc.ca) and approval by BEST. BIAST members are informed about the actual status of the mission via a monthly email newsletter. Last but not least, the BRITE-Constellation Ground-Based Observing Team (GBOT) provides a plat- 
form for BRITE scientists and observers worldwide to support collaborations and maximize the scientific output of BRITE-Constellation. The brightness of the targets facilitates ground-based support observations: even amateurs with modest-sized telescopes have obtained sciencequality high-resolution spectroscopy.

\section{First scientific results}

The first three scientific papers have been published in a short series [5], [8], [9], and have examined three different types of object in the Centaurus field. At this early stage in the mission, only the Austrian BAb and UBr were available in full science mode, and were later joined by BLb and BTr after their commissioning had been completed.

First, the brightest rapidly oscillating Ap (roAp) star $\alpha$ Circini was observed over 33 of its 4.479-d rotational periods. The blue and red filter light curves were found to be qualitatively different, with the blue light curve showing a single-wave variation, whereas the red light curve exhibited a double-wave structure. Both were analysed using Bayesian photometric imaging. In addition, the strongest roAp-type oscillation were recovered in both filters, demonstrating the photometric sensitivity of BRITE data. We refer to [8] for details and the full analysis.

Together with the brightest roAp star, the brightest $\beta$ Cephei star, $\beta$ Centauri, was studied during the same observing campaign. This triple system contains two pulsating B-type stars, one of them with a measured magnetic field. Both stars exhibit pressure and gravity modes. Previous studies of the system revealed two oscillation modes spectroscopically; photometric studies remained inconclusive. BRITE observations revealed a completely different picture. A total of 17 independent frequencies (8 likely g mode pulsations and $9 \mathrm{p}$ modes) and two combinations thereof were detected [5]. The orbital light-time effect may reveal which frequencies in the light variations originate from which star, but since the orbital period of the B-star binary is close to one year, more observations are needed to this end - and are certainly worth the effort.

The third of the initial three BRITE science publications reported a study of two Be stars, $\mu$ and $\eta$ Centauri [9]. Whereas in $\mu$ Cen, strong light echoes from the inner circumstellar disk, which is viewed face-on, prevented new insights into the pulsational properties, the study of $\eta$ Cen turned out to be more revealing. The modulation frequency of the star-disk mass transfer is equal to the frequency difference of two nonradial pulsation modes. This low frequency also modulates the (large) amplitude and the frequency of a circumstellar (so-called Štefl frequency), which is slightly lower than those of the stellar pulsations. The modulations in the mass loss are linked to variations in this Štefl frequency. This implies the presence of two engines causing the pulsation-mass loss interactions, a pulsational one from the star, and one related to viscosity in the circumstellar disk. In-depth arguments and discussions can be found in [9].

Another $\beta$ Cephei studied by BRITE is $v$ Eridani [7], observed in the Orion II field by BAb, BLb, BTr and BHr.
This star has been the subject of detailed observational and asteroseismic studies before, which have mostly concentrated on its p-mode spectrum. Earlier ground-based observations did detect two low-frequency g modes for $v$ Eridani, but it was the new BRITE observations that revealed six new gravity modes, firmly establishing it as a hybrid pulsator. The excitation of these $\mathrm{g}$ modes is still a problem for theoretical modelling. Whereas it is possible to excite a few $g$ modes in a certain frequency range in stellar models, reproducing the whole extent of the observed gravity mode domain is extremely difficult. To this end, opacity-modified models of $v$ Eridani were computed [11]; only those can account for the pulsational mode instability. On the other hand, such models also have significantly modified interior structure and it remains to be seen how realistic these modifications are. Future analysis of BRITE measurements of additional $\beta$ Cephei stars may provide an answer to this question.

When embarking on new types of observation, and achieving better precision than in the past, new scientific discoveries can result. Also BRITE has new discoveries to offer. In addition to the discovery of the role of difference frequencies in the mass loss of Be stars, BRITE has revealed the presence of massive heartbeat stars. These objects are close binaries in eccentric orbits, some of which also show tidally excited pulsations. The Kepler mission has discovered approximately two dozen such systems (e.g. [12]). However, all of these are composed of stars with masses below $2 \mathrm{M}_{\odot}$. With BRITE-Constellation, three more such systems have been discovered so far. $\iota$ Orionis [13] consists of an O9-type primary $\left(23 \mathrm{M}_{\odot}\right)$ and a B-type secondary $\left(13 \mathrm{M}_{\odot}\right)$, where the $\mathrm{O}$ star also shows tidally induced oscillations and is therefore the first of its kind. $\epsilon$ Lupi is the first doubly-magnetic massive star binary [14] with components of 8.7 and $7.3 \mathrm{M}_{\odot}$, respectively, and it shows peak flux modulation at periastron [15]. Finally, $\zeta$ Centauri $\left(M \approx 7 \mathrm{M}_{\odot}\right)$ also exhibits a light curve strongly indicative of periastron brightening [16]; new radial velocities have been obtained to confirm this hypothesis.

\section{Conclusions}

Being the first nanosatellite mission carrying out astrophysical research, BRITE-Constellation had to overcome many "childhood diseases", including initially unexpected radiation damage of its $\mathrm{CCD}$ detectors, interference issues impeding the communication between the satellites and the European-based ground stations, and systematic effects occurring in the light curves. However, the child has grown up and developed antibodies successfully fighting these diseases. The mission has been in full science operation for about two years and is now in its presumably most fruitful phase of data acquisition.

The BRITE project is open for collaboration that can be established in several ways, be it through the submission of target proposals (a call is open at the time of writing), be it through joining the BRITE International Advisory Team (BIAST) or the Ground-Based Observing Team 
(GBOT), or be it through attending the next BRITE science conference which will take place in Canada in August 2017.

For more information on the project itself, visit the BRITE home page http://www.brite-constellation.at/. Should you be interested in BRITE data, have a look at the BRITE photometry wiki (http://brite.craq-astro.ca/doku. php). In case you are a social media user, feel free to connect through the BRITE-Constellation facebook page.

\section{Acknowledgments}

The Polish contribution to the BRITE project is supported by the NCN grants 2011/01/B/ST9/05448, 2011/01/M/ST9/05914, 2011/03/B/ST9/02667 and 2015/18/A/ST9/00578. GAW and SMR acknowledge Discovery Grant support from the Natural Science and Engineering Research Council (NSERC) of Canada. AFJM is grateful for financial aid from NSERC and FQRNT (Quebec). KZ acknowledges support by the Austrian Fonds zur Förderung der wissenschaftlichen Forschung (FWF, project V431-NBL). BTr operations are supported through a Canadian Space Agency (CSA) Academic Development grant. RK and WW acknowledge financial support by the Austrian Space Application Programme (ASAP) of the Austrian Research Promotion Agency.

\section{References}

[1] Weiss W. W. et al., PASP 126, 573 - 585 (2014)

[2] Pablo H. et al., PASP 128, 125001 (20pp) (2016)

[3] Popowicz A., Proc. SPIE 99041R (2016)

[4] Popowicz A., et al., in preparation

[5] Pigulski A., et al., A\&A 588, A55 (2016)

[6] Buysschaert B., et al., A\&A, submitted

[7] Handler G., et al., MNRAS 464, 2249 (2017)

[8] Weiss W. W., et al., A\&A 588, A54 (2016)

[9] Baade D., et al., A\&A 588, A56 (2016)

[10] Bruntt H., et al., MNRAS 396, 1189 (2009)

[11] Daszyńska-Daszkiewicz J., et al., MNRAS 466, 2284 (2017)

[12] Thompson S. E., et al., ApJ 753, 86 (2012)

[13] Pablo H., et al., MNRAS, in preparation

[14] Shultz M., et al., MNRAS 454, L1 (2015)

[15] Wade G. A., et al., Polish Astronomical Society Proceedings, submitted

[16] Handler G., et al., in preparation 